\title{
A Hypothesis of Brain-to-Brain Coupling in Interactive New Media Art and Games Using Brain-Computer Interfaces
}

\author{
Polina Zioga ${ }^{1}$, Paul Chapman ${ }^{1}$, Minhua $\mathrm{Ma}^{2}$, and Frank Pollick ${ }^{3}$ \\ ${ }^{1}$ Digital Design Studio, Glasgow School of Art, Glasgow, UK \\ $\{$ P.Zioga1, P.Chapman\}@gsa.ac.uk \\ ${ }^{2}$ School of Art, Design and Architecture, University of Huddersfield, Huddersfield, UK \\ M.Ma@hud.ac.uk \\ ${ }^{3}$ School of Psychology, University of Glasgow, Glasgow, UK \\ Frank.Pollick@glasgow.ac.uk
}

This is the original author's accepted version of the manuscript, published by Springer on 28/05/2015. To cite this manuscript:

Zioga P., Chapman P., Ma M., Pollick F. (2015) A Hypothesis of Brain-to-Brain Coupling in Interactive New Media Art and Games Using Brain-Computer Interfaces. In: Göbel, S., Ma, M., Baalsrud Hauge, J., Oliveira, M.F., Wiemeyer, J., Wendel, V. (eds) Serious Games. JCSG 2015. Lecture Notes in Computer Science, vol 9090. Springer, Cham: 103-113. DOI: 10.1007/978-3-319-191263_9

The publisher’s version is available at: https://doi.org/10.1007/978-3-319-19126-3_9 


\title{
A Hypothesis of Brain-to-Brain Coupling in Interactive New Media Art and Games Using Brain-Computer Interfaces
}

\author{
Polina Zioga ${ }^{1}$, Paul Chapman ${ }^{1}$, Minhua Ma ${ }^{2}$, and Frank Pollick ${ }^{3}$ \\ ${ }^{1}$ Digital Design Studio, Glasgow School of Art, Glasgow, UK \\ \{P.Zioga1, P.Chapman\}@gsa.ac.uk \\ ${ }^{2}$ School of Art, Design and Architecture, University of Huddersfield, Huddersfield, UK \\ M.Ma@hud.ac.uk \\ ${ }^{3}$ School of Psychology, University of Glasgow, Glasgow, UK \\ Frank.Pollick@glasgow.ac.uk
}

\begin{abstract}
Interactive new media art and games belong to distinctive fields, but nevertheless share common grounds, tools, methodologies, challenges, and goals, such as the use of applications and devices for engaging multiple participants and players, and more recently electroencephalography (EEG)-based brain-computer interfaces (BCIs). At the same time, an increasing number of new neuroscientific studies explore the phenomenon of brain-to-brain coupling, the dynamics and processes of the interaction and synchronisation between multiple subjects and their brain activity. In this context, we discuss interactive works of new media art, computer and serious games that involve the interaction of the brain-activity, and hypothetically brain-to-brain coupling, between multiple performer/s, spectator/s, or participants/players. We also present Enheduanna - A Manifesto of Falling (2015), a new live brain-computer cinema performance, with the use of an experimental passive multi-brain BCI system under development. The aim is to explore brain-to-brain coupling between performer/s and spectator/s as means of controlling the audio-visual creative outputs.
\end{abstract}

Keywords: brain-to-brain coupling, brain-computer interface (BCI), humancomputer interaction (HCI), electroencephalography (EEG), new media art, computer games, serious games, performer, audience, spectator, participant, player.

\section{Introduction}

Although interactive works of new media art, computer and serious games belong to distinctive fields, they also share common grounds, tools, methodologies, challenges, and goals. Their development and implementation is based on the use of computer software, like programmes for audio-video creation and 3d animation. They seek to communicate a message to the spectators/participants/players, help them achieve a level of understanding of an idea/concept and motivate them to reflect upon notions. They search for optimum ways to inspire them, in order to pursue specific tasks during 
a performance, inside an environment (physical, digital or virtual) or during a game, interact with the work/game and each other and through this interaction to achieve a meaningful engagement. The result of this engagement might be a new perception and/or exercise of a new attitude, that in the case of serious games could also have an educational or health benefit.

Nowadays, in both fields the use of different applications and devices for engaging multiple participants and players is highly disseminated and rapidly increasing, from the use of mobile applications, to human-computer interaction devices and after 2007, electroencephalography (EEG)-based brain-computer interfaces (BCIs). The development of new low-cost wireless devices has radically changed the use of BCIs in the arts, which originates in the 1960s [1], and alongside the research on applications for populations suffering from neurological deficiencies and disabilities. At the same time, it has enabled the development of the first computer games and applications, mainly for entertainment, and more recently serious games.

In a parallel course with the brain-computer interfaces' advancements and breakthroughs, in the fields of neuroscience and experimental psychology has emerged a new and increasing interest in studying the mechanisms, dynamics and processes of the interaction and synchronisation between multiple subjects and their brain activity. Hasson et al. called in 2012 "for a shift from a single-brain to a multi-brain frame of reference", arguing that "in many cases the neural processes in one brain are coupled to the neural processes in another brain via the transmission of a signal through the environment $[\ldots]$ leading to complex joint behaviors that could not have emerged in isolation." [2].

In this context, after a brief introduction in section 1 to brain-to-brain coupling, we discuss in section 2 interactive works of new media art, computer and serious games that involve the interaction of the brain-activity and hypothetically brain-to-brain coupling between performer/s, spectator/s, and multiple participants/players. And in section 3 we present a new live brain-computer cinema performance with the use of an experimental passive multi-brain BCI system under development. The aim is to explore brain-to-brain coupling between the performer/s and member/s of the audience as means of controlling the audio-visual creative outputs.

\subsection{Brain-to-brain coupling}

Hasson et al. [2] analogised brain-to-brain coupling to a wireless communication system, "in which two brains are coupled via the transmission of a physical signal (light, sound, pressure or chemical compound) through the shared physical environment. [...] The coordination of behavior between the sender and receiver enables specific mechanisms for brain-to-brain coupling unavailable during interactions with the inanimate world."

The authors continue explaining how the exchange of information between two individuals bears similarities to the transmission of information between two areas of a single brain. An example is the coupling and the enhancement of the signal-to-noise ratio of the frequency of the speech with the auditory cortical oscillations that have a similar frequency [2], which can also be extended and further amplified with the 
presence of visual information and stimuli, like watching the speaker's face and lips. Relevant studies include functional Magnetic Resonance Imaging (fMRI) scanning of both speakers and listeners during natural verbal communication, which have shown that "the speaker's activity is spatially and temporally coupled with the listener's activity". The listener's brain activity on average mirrors the speaker's activity with a delay, but there are also areas that exhibit predictive anticipatory responses and in fact "the greater the anticipatory speaker-listener coupling, the greater the understanding" [3]. Other experiments have shown that performing and observing hand gestures and facial expressions can also result in brain-to-brain coupling [4] and related results have been obtained with experiments that involved facial communication of affect [5]. The majority of these studies investigate brain-to-brain coupling through the use of intersubject correlation (ISC), which is "a measure of how similar subjects' brain activity is over time", and is also considered highly reliable, allowing "the exploration of sensory areas involved in natural viewing of long stimulus segments i.e. $>6$ min." [6].

The phenomenon and theory of brain-to-brain coupling is not only innovative for the fields of neuroscience and psychology, but the potential applications in the frame of multi-brain interactive works of new media art, computer and serious games is apparent and has already attracted the attention of researchers, artists and developers.

\section{From interacting with interfaces to interactive brains}

In this section we will discuss representative examples of interactive works of new media art, computer and serious games, where the brain activity of two or more performers, spectators, participants or players is monitored and processed jointly.

\subsection{In interactive new media art}

Amongst artists and performers the notion of communicating and establishing a feeling of being connected with each other and the audience is not new, but rather part of their anecdotal experience. However, in recent years with the advancement of neurosciences and the new EEG technology they managed to realise works and projects as a manifestation of their inner subjective experiences. Although to our present knowledge, artists so far have not explicitly addressed brain-to-brain coupling as a concept in their work, they have implemented related ideas, such as coherence and synchronisation between multiple participants or between performer/s and spectator/s.

Mariko Mori's Wave UFO (2003) is one of the first examples, an immersive video installation, where computer-generated graphics are not only combined with the "realtime interpretation of three participants' alpha, beta, and theta brain-waves”. They are also used as a visualisation of the synchronisation between the alpha-wave activity of each participant's brain hemispheres with a pair of small spheres, the "Coherence Spheres" [7]. When synchronisation is achieved, the "Coherence Spheres" are joining together, and when all three participants reach this state, a circle is created. 
More recently, a series of projects, like Measuring the Magic of Mutual Gaze (2011), The Compatibility Racer (2012) and The Mutual Wave Machine (2013), by the Marina Abramovic Institute Science Chamber and the neuroscientist Dr. Suzanne Dikker, explore "moments of synchrony" of the brain-activity between two participants, when they interact by gazing at each other [8]. As Dikker explains by "moments of synchrony" are meant points in time when the two participants present the same predominant brain-activity [9]. In both cases, Mariko Mori's work and the projects by the Marina Abramovic Institute Science Chamber and Dr. Suzanne Dikker, the concept of synchronisation between multiple participants is implemented by focusing in and examining the temporal EEG brain-activity within the range of either one specific frequency band and/or examining a wider spectrum.

In the field of live computer music performances, Eaton, Jin, and Miranda presented in 2014 the piece The Space Between Us, where the brainwaves of a singer and a member of the audience are measured and processed in real-time separately or jointly, as an attempt of bringing the "moods of the audience and the performer closer together" [10]. In this case the phenomena investigated, are the levels of valence and arousal levels within the EEG brain-activity [11].

\subsection{In computer and serious games}

The use of BCIs in computer and serious games is a new trend and as recent as the development of the new low-cost commercial devices. There are different approaches in the implementation of BCIs in games, such as the use of neurofeedback, visually evoked potentials, and motor imagery, while the BCI itself is not always the central game mechanic. In any case though, the majority of the games are designed for the interaction of one player's brain activity. A smaller number, the "multi-brain games" [12], involve the interaction of two or more players' brain-activity, not necessarily at the same time, while they are most commonly designed for multi-brain competition and less often for multi-brain collaboration. However, brain-to-brain coupling seems to be promoted more in conditions of collaboration, than in conditions of competition. A game of this kind, designed for research purposes, is Mind the Sheep! (MTS!), which allows "both BCI and non-BCI play" with the use of an EEG cap, and it is designed for a single-user, but also multi-users either collaborating or competing. The players use $\mathrm{BCI} / \mathrm{s}$ in order to select and move dogs that help them fence the sheep in, while they can collaborate through visual, vocal and gestural communication [12]. Another similar game is BrainArena, a football game for two players with two BCIs. The users "can score goals on the left or right of the screen by simply imaging left or right hand movements”. They can play either by competing against each other or by collaborating, in which case their brain activities are combined. The results of the experiments conducted for the evaluation of the performance and the user experience, have interestingly suggested that the multi-user conditions can be "operational, effective, and more engaging" for the players, and even more, some of them showed significantly improved performance comparing to the single-user condition [13]. Observations like these can open a dialogue with behavioural studies, which can further advance the field. For example Bahrami et al. studied collective decision making between different 
observers. Their results showed that for "two observers of nearly equal sensitivity" collective decision making was more efficient than a single decision making process, "provided that they were given the opportunity to communicate freely, even in the absence of any feedback about decision outcomes." However, for observers with very different sensitivities the collaborative outcome was worse than the single decision making process [14].

Another example of brain-to-brain communication and synchronisation, applied in a computer game, is the first direct brain-to-brain interface (BBI) between two humans, demonstrated by Rao et al. The interface, which is non-invasive, is designed to detect motor imagery in the EEG signals recorded from one participant (the "sender"), which are then transmitted over the internet and delivered to the motor cortex of a second participant (the "receiver") with the use of transcranial magnetic stimulation (TMS). The BBI is used in order for the participants to corporate and achieve a desired goal in a computer game, which was to "defend a city [...] from enemy rockets fired by a pirate ship" with the use of a cannon. More specifically, the "sender" was able to see the game on a computer screen, but could not control the cannon. No input device was provided, but the participant could communicate his intent to fire by imaging right hand movement. Through the recording of his EEG signals a cursor was controlled. When the cursor hit "fire", a signal was transmitted from his computer over the internet, to the computer connected to the TMS machine, which was then sending a pulse to the "receiver". The "receiver" could not see the game, but the stimulation he/she received was causing a quick movement of the right hand, enabling him/her to press a touchpad, in order to fire the cannon. The two participants were remotely located and had no communication with each other, apart from the BBI [15].

The above examples strongly indicate the potential of the use of "multi-brain games" not only for entertainment, but also in the context of applications for educational and health purposes. Indeed, following the trend that has already emerged, the first serious games for single-brain player have been released, and we expect in the near future also the development of the first serious games for multi-users. A relevant announcement was made recently by MyndPlay, which released the game "Focus Pocus", described by the company as an "Interactive attention and brain development training game for children” [16], and is designed for educational purposes as well as a supplementary treatment method for Attention Deficit Hyperactivity Disorder (ADHD). The game can be played by both a single-player, as well as multiple-players.

\section{Enheduanna - A Manifesto of Falling: a live brain-computer cinema performance}

Enheduanna - A Manifesto of Falling (2015), ${ }^{1}$ directed by Polina Zioga [17], is a new work which falls under the definitions of live brain-computer mixed-media

1 The title and the main theme of the performance are taken from the historical figure of Enheduanna (са. 2285-2250 B.C.E.), an Akkadian Princess and High Priestess in the 
performances, that combine live, mediatized representations and the use of BCIs [1], and live cinema, which is "[...] real-time mixing of images and sound for an audience, where $[\ldots]$ the artist's role becomes performative and the audience's role becomes participatory." [18]. The performance, with an approximate duration of 50 minutes, involves the live act of three performers, a live visuals (live video projections) and BCI performer, a live electronics and music performer, an actress, and the participation of at least one member of the audience, with the use of an experimental passive multibrain EEG-based BCI system we are currently developing at the Digital Design Studio (DDS), Glasgow School of Art.

\subsection{The creative and cognitive approach}

The performer/s' activity and the participatory role of member/s of the audience are enhanced and characterised by the use of their real-time brain-activity as a physical expansion of the creative process, as an act of co-creating and co-authoring, and as an embodied form of improvisation, which is mapped real-time to the visual (live projections) and/or audio (live electronics) outputs. Additional elements borrowed by the practices of live cinema include the use of non-linear narration and storytelling approach through the fragmentation of the image, the frame and the text, resulting in a greater freedom concerning the manipulation of time and the "start-middle-end" structure.

Therefore the experience and engagement of the audience is multi-dimensional and bears analogies to free viewing for example of films, which is extensively studied in neurocinematics, an interdisciplinary field investigating the effect of films on the spectators' brain activity, searching for similarities in their spatiotemporal responses [19]. In the case of Enheduanna - A Manifesto of Falling (2015) live brain-computer cinema performance, our aim is double: the development of a multi-brain EEG-based BCI system, which will enable the use of the brain activity of one performer and at least one member of the audience as a creative tool, but also as a tool for investigating the passive multi-brain interaction between them. We endeavour to find potential evidence of temporal real-time brain-to-brain coupling, which to our knowledge will be the first time under the specific conditions.

\subsection{The passive multi-brain EEG-based BCI system}

By "passive” BCI we imply that the outputs are derived from "arbitrary brain activity without the purpose of voluntary control", in contrast to "active" BCIs where the outputs are derived from brain activity "consciously controlled by the user", and the "reactive" BCIs who derive their outputs from "brain activity arising in reaction to external stimulation" [20].

The design of the passive multi-brain EEG-based BCI system consists of two main parts and it is based on a model of interactions between the performer/s and the audience

Sumerian city of Ur (present-day Iraq), who is regarded as possibly the first known author and poet in the history of human civilisation, regardless of gender. 
in the context of live brain-computer mixed-media performances (figure 1). The model demonstrates the collective participation and co-creation of the mediatized elements of the performance.

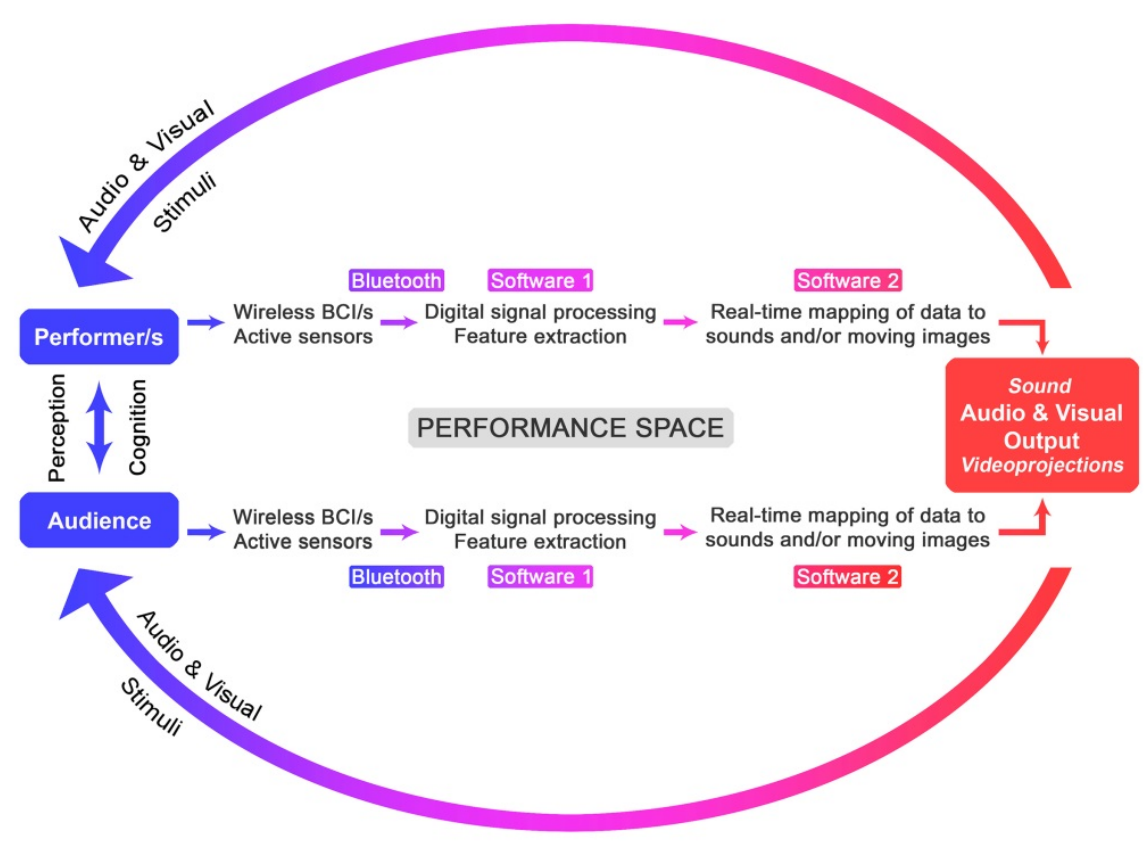

Fig. 1. A model of interactions between the performer/s and the audience in live brain-computer mixed-media performances. Adapted from Zioga et al. [1].

The first part of the system, involves the use of commercial grade EEG-based wireless devices, and more specifically the MyndPlay BrainBandXL EEG Headset, which has two dry sensors with one active located in the prefrontal lobe (Fp1) [21]. The components of the device are presented in figure 2; the headband on which the rest of the components are attached, the unit that transmits via Bluetooth the EEG data to the computer, the USB cable for charging the unit, the two dry sensors attached to a soft material similar to the one used for the headband, and the ear clip with the grounding electrode for the ear lobe. 


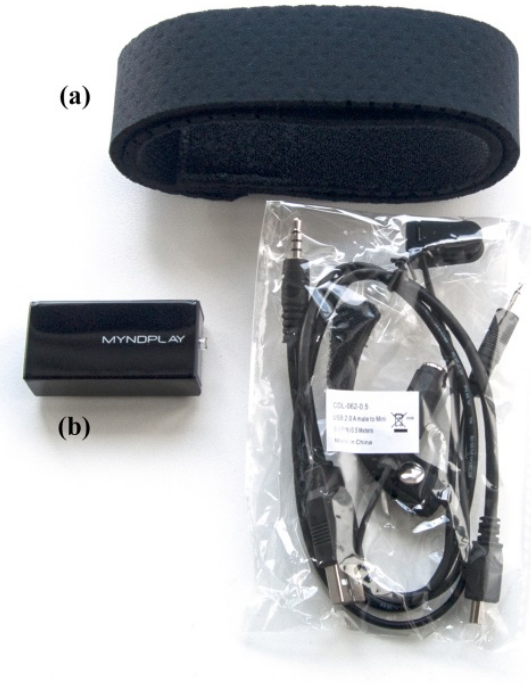

(c)

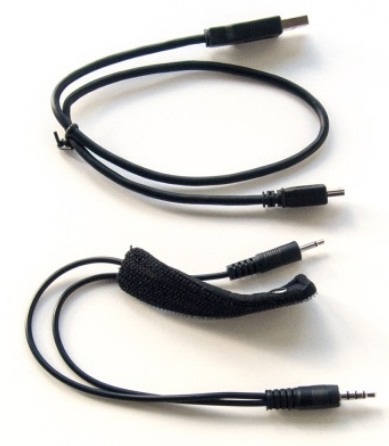

(e)

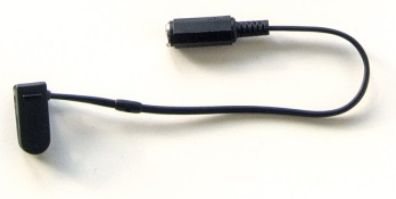

Fig. 2. The components of the MyndPlay BrainBandXL EEG Headset: (a) the headband on which the rest of the components are attached, (b) the unit that transmits via Bluetooth the EEG data to the computer, (c) the USB cable for charging the unit, (d) the two dry sensors attached to a soft material similar to the one used for the headband, and (e) the ear clip with the grounding electrode for the ear lobe.

The first part of the system design involves also the real-time extracting and processing of the raw EEG signals from each participant and device, which are transmitted wirelessly to a computer via Bluetooth, and are collected and processed with OpenViBE software [22] by using algorithms that follow the frequency analysis method. Regarding the processing methodology, the focus is on the oscillatory processes of the performer/s and the member/s of the audience brain activity. The model is dynamic, meaning that the output is depending/changing according to time and it is also causal, meaning that the output depends only on the parameters in specific time and is not able to look/predict into the future [23]. With the use of band-pass filters, the $4-40 \mathrm{~Hz}$ frequencies, that fall within the spectrum of theta $(4-7 \mathrm{~Hz})$, alpha $(8-13 \mathrm{~Hz})$, beta $(14-25 \mathrm{~Hz})$, and lower gamma $(25-40 \mathrm{~Hz})$ frequency bands, and are meaningful in the conditions of the performance, are selected. The $<4 \mathrm{~Hz}$ frequency, which corresponds to the delta band, and is associated with deep sleep, is rejected, in order to suppress low pass noise, electrooculographic (EOG) and electrocardiographic (ECG) artifacts (artifacts deriving from the movements of the eyes and the cardiac muscle respectively). Also the $40 \mathrm{~Hz}$ and above frequencies are rejected, in order to supress electromyographic (EMG) artifacts from the body muscle movements, high pass and line noise from electrical devices in the proximity of the performer/s and the participating spectator/s. The processing continuous with static and moving average filters in order to compute and output at any given time the power of each selected 
frequency band and in this way to identify the predominant one. Moreover, the processed EEG data of each participant are being recorded, not only as documentation of the performer/s' and the participating spectator/s' brain-activity, but also in order to be analysed off line after the performance and compare them with related studies conducted in a lab environment.

The second part of the system design is the transfer of the collective processed data to a second software, like Pd-extended and MAX/MSP, in order to map the values of the performer/s and the spectator/s brain-activity, both separately and jointly, to the visual and/or audio creative outputs of the performance. In this way, we attempt to make physically apparent and visualise in a meaningful way the predominant underlying ongoing processes and maybe a potentially achieved temporal brain-to-brain coupling.

The performance with the under development system described here is due to be presented in the coming months and the results will be published accordingly.

\section{Discussion}

The phenomenon of brain-to-brain coupling, the related theories and studies have already set a new horizon in neuroscience and psychology for the understanding of human intersubject communication and interaction. Together with the accelerating advances in biomedical engineering, computer science and the continuous improvement of modern brain-computer interface design, new possibilities are emerging for novel applications. Art and entertainment are undoubtedly at the forefront of imagination and creativity. Today artists, creative practitioners and designers have not only been enabled to use their own brain in their creative practices in the most direct way made so far possible, but they are also given a new opportunity to manifest the real-time brain-activity interaction between the performers, their audience, multiple participants and players. What has already been demonstrated during neuroscientific lab experiments is yet to be proven in the context of interactive works of new media art, computer and serious games. This will also change the map of sharing experiences, co-creating and co-authoring, but it has also great potential for feeding back neuroscience and psychology by proposing novel ways of multi-brain engagement, with potentially educational and health benefits. Of course we are still at the beginning and there are multiple questions arising. What kind of methods need to be developed and what tools to be used in order to investigate brain-to-brain coupling in the context of interactive works of new media art, computer and serious games? Are there specific conditions and cognitive tasks that could be proven more appropriate? What might be the influence of the environment, physical, digital or virtual, where the multi-brain interaction is taking place? What role can the difference in the sensitivity of different subjects play? We hope that the experience and knowledge gained from the live braincomputer cinema performance outlined in this paper will contribute to this discourse, and we expect in the future more answers, questions and new innovative applications to emerge. 
Acknowledgements. P.Z. is supported by the Global Excellence Initiative Fund PhD Studentship awarded by the Glasgow School of Art. Enheduanna - A Manifesto of Falling live brain-computer cinema performance is due to be presented at the Centre for Contemporary Arts in Glasgow, on the $30^{\text {th }}$ and $31^{\text {st }}$ of July 2015, with the support of NEON Organization's 2014-2015 Grant for Performance Production.

\section{References}

1. Zioga, P., Chapman, P., Ma, M., and Pollick, F.: A Wireless Future: performance art, interaction and the brain-computer interfaces. In: INTER-FACE: International Conference on Live Interfaces 2014. Lisbon (2014).

2. Hasson, U., Ghazanfar, A.A., Galantucci, B., Garrod, S., and Keysers, C.: Brain-to-brain coupling: a mechanism for creating and sharing a social world. Trends in Cognitive Neuroscience, 16(2), pp.114-121. doi:10.1016/j.tics.2011.12.007 (2012).

3. Stephens, G.J., Silbert, L., and Hasson, U.: Speaker-listener neural coupling underlies successful communication. In: Proceedings of the National Academy of Sciences of the United States of America, 107(32), pp.14425-14430 (2010).

4. Schippers, M. B., Roebroeck, A., Renken, R., Nanettia, L., Keysers, C.: Mapping the information flow from one brain to another during gestural communication. In: Proceedings of the National Academy of Sciences of the United States of America, 107(20), pp.93889393 (2010).

5. Anders, S., Heinzle, J., Weiskopf, N., Ethofer, T., and Haynes, J.-D.: Flow of affective information between communicating brains. Neuroimage, 54(1-4), pp.439-446 (2011).

6. Jola, C., McAleer, P., Grosbras, M.-H., Love, S.A., Morison, G., and Pollick, F.E.: Uni- and multisensory brain areas are synchronized across spectators when watching unedited dance recordings. i-Perception, 4(4), pp. 265-284 (2013).

7. Mori, M., Kunsthaus Bregenz, and Schneider, E.: Mariko Mori: wave UFO. Verlag der Buchhandlung Walther König, Köln (2003).

8. Dikker, S.: REAL-TIME INTERACTIVE BRAIN INSTALLATIONS (Last Modified September 2014). Available from: https://files.nyu.edu/sd1083/public/art.html [Accessed October 19, 2014].

9. Marina Abramovic Institute: Out of the Lab (2014). Available from: http://www.immaterial.org/content/2014/6/9/out-of-the-lab [Accessed October 19, 2014].

10. Eaton, J.: The Space Between Us. Vimeo video, 06:35. Posted by “joel eaton” (2015). Available from: https://vimeo.com/116013316 [Accessed March 04, 2015].

11. Eaton, J., Jin, W., and Miranda, E.: The Space Between Us: A Live Performance with Musical Score Generated via Affective Correlates Measured in EEG of One Performer and an Audience Member. In: NIME'14 International Conference on New Interfaces for Musical Expression, pp.593-596. NIME ‘14, London (2014).

12. Nijholt, A. and Gürkök, H.: Multi-Brain Games: Cooperation and Competition. In: Stephanidis, C. and Antona, M. (eds.) Universal Access in Human-Computer Interaction Design Methods, Tools, and Interaction Techniques for eInclusion, part 1, pp. 652-661. Springer Heidelberg Dordrecht London NewYork (2013).

13. Bonnet, L., Lotte, F., and Lecuyer, A.: Two Brains, One Game: Design and Evaluation of a Multi-User BCI Video Game Based on Motor Imagery. IEEE Transactions on Computational Intelligence and AI in games, IEEE Computational Intelligence Society, 5(2), pp.185-198 (2013). 
14. Bahrami, B., Olsen, K., Latham, P.E., Roepstorff, A., Rees, G., and Frith, C.D.: Optimally interacting minds. Science, 329(5995), pp.1081-1085 (2010).

15. Rao, R.P.N., Stocco, A., Bryan, M., Sarma, D., Youngquist, T.M., Wu, J., and Prat, C.: A Direct Brain-to-Brain Interface in Humans. University of Washington Computer Science and Engineering. Technical Report No. UW-CSE-14-07-01. July, 2014. Available from: http://homes.cs.washington.edu/ rao/brain2brain/UW-CSE-14-07-01.PDF.pdf [Accessed October 22, 2014].

16. MyndPlay: Focus Pocus for Windows (2015). Available from: http://myndplay.com/software.php?swid=19 [Accessed March 04, 2014].

17. Zioga, P., and Katsinavaki, A.: Enheduanna - A Manifesto of Falling. Premiere 30th July 2015. Centre for Contemporary Arts, Glasgow, UK. Performers: Anastassia Katsinavaki, Minas Borboudakis, and Polina Zioga. Director: Polina Zioga. (2015).

18. Willis, H.: Real Time Live: Cinema as Performance. AfterImage, 37(1), pp. 11-15 (2009).

19. Hasson, U., Landesman, O., Knappmeyer, B., Vallines, I., Rubin, N., and Heeger, D.: Neurocinematics: The Neuroscience of Film. Projections: The Journal for Movies and Mind, 2(1), 1-26 (2008).

20. Zander T.O., Kothe C, Welke S, and Roetting M.: Enhancing human-machine systems with secondary input from passive brain-computer interfaces. In: Proceedings of the 4th International BCI Workshop \& Training Course, Graz, Austria, 2008. Graz University of Technology Publishing House, Graz (2008).

21. MyndPlay: BrainBandXL \& MyndPlay Pro Bundle (2015). Available from: http://myndplay.com/products.php?prod=9 [Accessed March 04, 2014].

22. Renard, Y., Lotte, F., Gibert, G., Congedo, M., Maby, E., Delannoy, Bertrand, V. O., and Lécuyer, A.: OpenViBE: An Open-Source Software Platform to Design, Test and Use BrainComputer Interfaces in Real and Virtual Environments, Presence : teleoperators and virtual environments, 19(1), (2010).

23. Swartz Center of Computational Neuroscience - University of California San Diego: Introduction To Modern Brain-Computer Interface Design Wiki (2012). Available from: http://sccn.ucsd.edu/wiki/Introduction_To_Modern_Brain-Computer_Interface_Design [Accessed 24 September, 2014]. 